\title{
Electron-beam deposition of metals for mask repair
}

\author{
${ }^{1}$ Young Choi and ${ }^{1,2}$ David C Joy* \\ ${ }^{1}$ EM Facility, University of Tennessee, Knoxville, TN 37996-0840, and \\ ${ }^{2}$ *ak Ridge National Laboratory, Oak Ridge, TN 37831-6064
}

The controlled deposition of metals resulting from the passage of an ion beam through an atmosphere of a suitable precursor gas is a well-established procedure for micro scale materials manipulation. While the ion technique is rapid and reliable it has the disadvantage that the beam itself can stain and contaminate the target with Gallium or other materials, and ion optics are less widely accessible than electron optical columns. We have therefore been investigating the theory and practice of depositing metal using only an electron beam and variety of gases. The aim of this work is to develop techniques that can be applied to the correction and repair of the optical, UV and EV masks used in lithography.

Deposition has been done in a Hitachi S3500N VPSEM, which has capable of 1 to $30 \mathrm{Kev}$ beam energy, and can sustain up to 300Pa in the specimen chamber. Gas was injected into the SEM chamber through a wobble stick controlled system equipped with four nozzles. Precursor gas is injected to $1 \mathrm{~mm}$ above the sample by manually controlled from outside of SEM through the needle. The deposition process is monitored using the EDS system attached to the microscope. From an analysis of the spectra produced the thickness of the deposition can be measured with good precision using calibration curves derived from Monte Carlo simulations. The contents are monitored with a residual gas analyzer, which reads out both the total chamber pressure as well as the partial pressure of the chosen precursor gas. ${ }^{[1]}$

In the work described here we study the deposition of the tungsten on to wafers of silicon or germanium employing $\mathrm{W}(\mathrm{CO})_{6}$ as precursor. Because tungsten hexa-carbonyl is a solid state at room temperature the container in which it is placed, and the delivery tube to the gas jet, must be maintained at a temperature high enough to ensure sublimation and eliminate condensation, but low enough to avoid decomposition. Figure (1) shows some test structure deposited at beam energy of $5 \mathrm{Kev}$ and current of below $1 \mathrm{nA}$ using $\mathrm{W}(\mathrm{CO})_{6}$ pressure in the range 0.1 to $20 \mathrm{~Pa}$. The thickness, as well the composition of the deposited materials was analyzed. Figure (2) shows an example of the variation of the X-ray spectrum during deposition onto germanium. Based on this X-ray spectrum, we can calibrate the thickness of the deposited area using the Monte-Carlo simulation. Figure (3) shows change of tungsten thickness relative to ratio of germanium and tungsten signal and figure (4) shows how the thickness of tungsten layer varies as function of time (or dose) for a specified beam current and dose rate. With above data, we can figure out roughly that the growth rate condition as the order of 1 tungsten atom per 1000 incident electrons, equivalent to a rate of 1 picogram of metal per each 2 microcoulombs of deposited charge.

The presence of electrostatic fields caused by charging induced in the sample, will result in ion drift, which may cause asymmetries in the deposition profile, and maintaining temperature is important for gas inlet. However, in tool such as the VPSEM, surface charging can be controlled or eliminated by choosing the pressure and composition of the gas atmosphere within the specimen chamber without compromising the deposition chemistry. ${ }^{[2],[3]}$ 


\section{Acknowledgements}

[1] The authors are grateful to Prof. P.D. Rack (UTK) for valuable suggestions and discussions.

[2] This work was supported by SRC contract LJ-962.003.

[3] Oak Ridge National Laboratory is managed by UT-Batelle, LLC for the USDOE under contract DE-AC05-00OR22725.

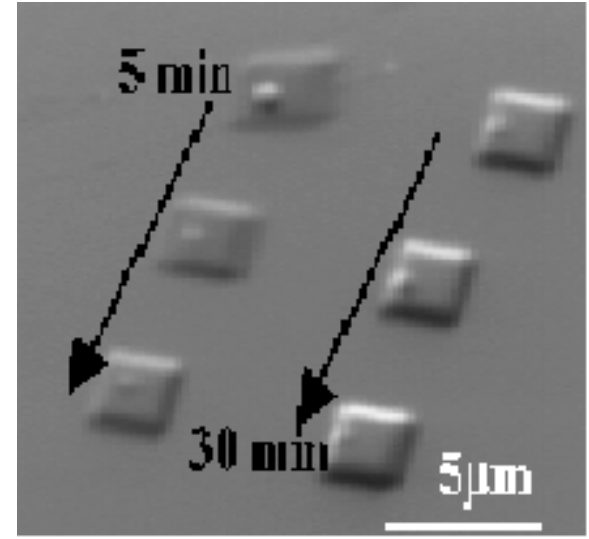

Fig. (1) SEM micrograph of tungsten deposited on a Ge wafer with $5 \mathrm{Kev}$ beam energy, $0.4 \mathrm{nA}$ beam current, scanned area $2.5 \times 2.5 \mu \mathrm{m}, 10 \mathrm{~Pa}$ of $\mathrm{W}(\mathrm{CO})_{6}$ precursor, scanned for 5 to 30 minutes.

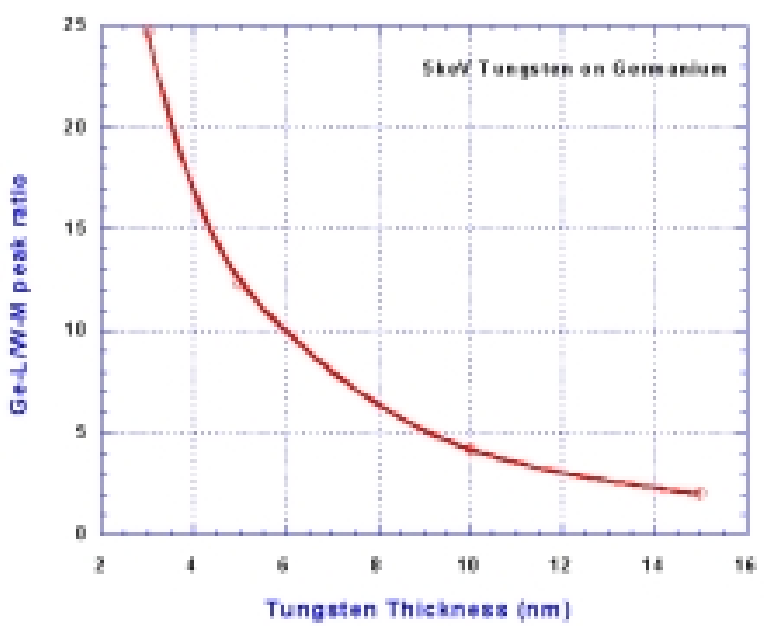

Fig. (3) The computed calibration curve for analysis at $5 \mathrm{Kev}$ beam energy showing the ratio of $\mathrm{W}$-m line to Ge-L line as a function of the thickness of the tungsten layer.

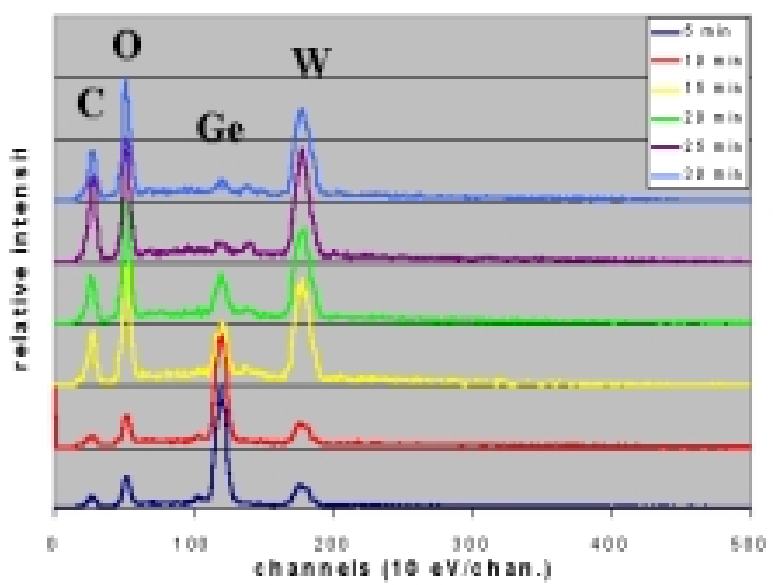

Fig. (2) A time sequence of EDS spectra collected at 5 minute intervals during the 30 minute deposition of fig. (1) showing the rise of $\mathrm{W}$ and the fall in the Ge signal.

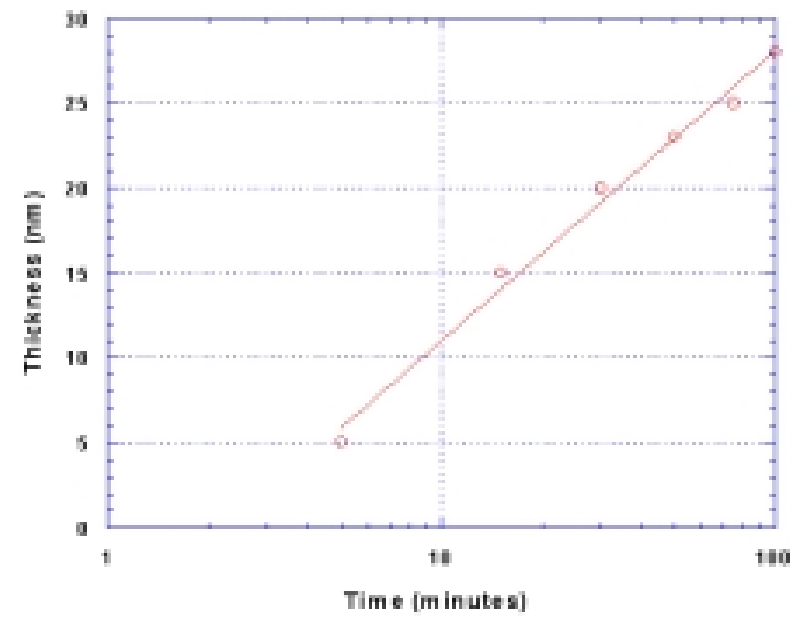

Fig. (4) Measured variation of thickness of deposited tungsten layer as a function of time using the condition of Fig. (1) 
https://doi.org/10.1017/S1431927603444929 Published online by Cambridge University Press 